\title{
NOUVELle
}

\section{Régulation épigénétique de la pluripotence chez l'embryon de souris}

Jérôme Collignon, Aitana Perea-Gomez
Département de Biologie du développement,

Institut Jacques Monod, CNRS, Université Paris 7,

Université Paris 6, 2, place Jussieu, Tour 43,

75251 Paris Cedex 05 France.

collignon@ijm.jussieu.fr
> Une étape importante pour la compréhension du rôle des modifications épigénétiques de la chromatine dans la régulation de la pluripotence des cellules de l'embryon de mammifère a été franchie avec une étude publiée récemment dans le journal Nature par l'équipe de M. Zernicka-Goetz [1]. Les bases de ce travail avaient été posées en 2005 avec la publication par cette même équipe d'une étude établissant qu'en fonction de l'orientation et de la séquence des clivages qui leur donnent naissance, les blastomères composant l'embryon de souris au stade 4 n'ont pas les mêmes capacités développementales, et que les destins de leur descendance peuvent être drastiquement différents [2].

Le premier plan de clivage du zygote, environ vingt-quatre heures après la fécondation, passe par son axe animalvégétal (AV), marqué au pôle animal par la présence du second globule polaire. Ce type de clivage est dit méridional (M). Le clivage de chacun des deux blastomères résultant peut être soit méridional, soit équatorial $(\varepsilon)$, c'est-à-dire perpendiculaire à l'axe AV (Figure IA). Ces deux blastomères ne se divisent pas en même temps et l'embryon passe donc par un stade intermédiaire où il ne compte que trois blastomères.

Au stade 4, trois morphologies embryonnaires sont distinguées, que l'on peut décrire comme tétraédrique, plane et en étoile (Figure 1A). Des études de lignage, après marquage d'une cellule au stade 2 blastomères, ont permis de comprendre leur origine [3]. La majorité ( $81 \%$ ) des embryons au stade 4 ont une morphologie tétraédrique qui résulte d'une séquence de clivage $M E$ ou EM. Les embryons dont la morphologie est en étoile ( $11 \%$ ) sont le résultat d'une séquence $M M$, les embryons dont la morphologie est plane (8\%), d'une séquence $\varepsilon \varepsilon$. Il avait été noté précédemment que pour une proportion importante des embryons, la descendance du premier blastomère à s'être divisé contribue de façon majoritaire à la région embryonnaire du blastocyste, comprenant la masse cellulaire interne $(\mathrm{MCl}$, dont les descendants formeront les tissus fœtaux) et le trophectoderme polaire (tissu extra-embryonnaire). Dans ce cas, la descendance du deuxième blastomère contribue préférentiellement à la région $a b$-embryonnaire, comprenant la partie de la $\mathrm{MCl}$ bordant la cavité du blastocèle et le trophectoderme mural. Cependant, ce type de répartition dépend très étroitement de la façon dont les deux premiers blastomères se sont divisés et ne représente pas le schéma général pour tous les embryons. De façon remarquable, il a été montré que c'est seulement dans le cas d'une séquence de clivage $M \varepsilon$ qu'il est possible de prédire la future polarité du blastocyste (Figure $1 B$ ). Dans le cas d'un clivage $E M$, la descendance du premier blastomère a des chances égales de contribuer soit à la région embryonnaire, soit à la région $a b$-embryonnaire du blastocyste. Pour les séquences de clivage MM et $\varepsilon \varepsilon$, l'allocation des cellules descendant des deux premiers blastomères n'est pas restreinte à l'une ou l'autre de ces régions.

Les embryons ME sont donc les seuls permettant de prédire le destin de chacun des quatre blastomères les constituant. Des expériences d'agrégation ont permis de préciser les capacités développementales de chacun de ces blastomères [2]. Le blastomère $e 2$, résultant de la division $\varepsilon$ et héritant de la majorité du cytoplasme de la région végétative, contribue principalement au trophectoderme mural, et ne permet que rarement d'obtenir un embryon viable lorsqu'il est agrégé à trois autres cellules d'origine identique (Figure 1C). En revanche, l'agrégation de blastomères $m$, issus de la division $M$, permet d'obtenir avec un taux comparable aux contrôles le développement à terme des embryons chimères résultants. II semble donc que ces blastomères diffèrent dans leur capacité à permettre le développement d'un embryon, ce qui suggère le partage inégal entre eux d'une information ou d'une activité préexistante.

Les marques épigénétiques qui décorent la chromatine ont souvent été proposées comme étant susceptibles de véhiculer une information importante pour la régulation de la pluripotence des cellules embryonnaires. Tirant parti de leur connaissance approfondie des séquences de clivage chez l'embryon de souris, Torres-Padilla et al. se sont intéressés à la distribution au stade 4 de trois modifications de l'histone $\mathrm{H} 3$, résultant de la méthylation d'arginines aux 
positions 2, 17 ou 26. L'utilisation d'anticorps spécifiques pour chacune de ces modifications a permis leur quantification relative au sein des noyaux d'un même embryon [1]. II a ainsi été montré que pour une fraction des embryons au stade 4 , le taux de méthylation n'est pas le même dans chaque blastomère. Les contrastes les plus élevés étant obtenus pour la modification H3R26me, c'est en suivant celle-ci que les investigations se sont poursuivies. TorresPadilla et al. ont démontré que ce sont les blastomères des embryons $M E$ qui présentent les variations les plus importantes,

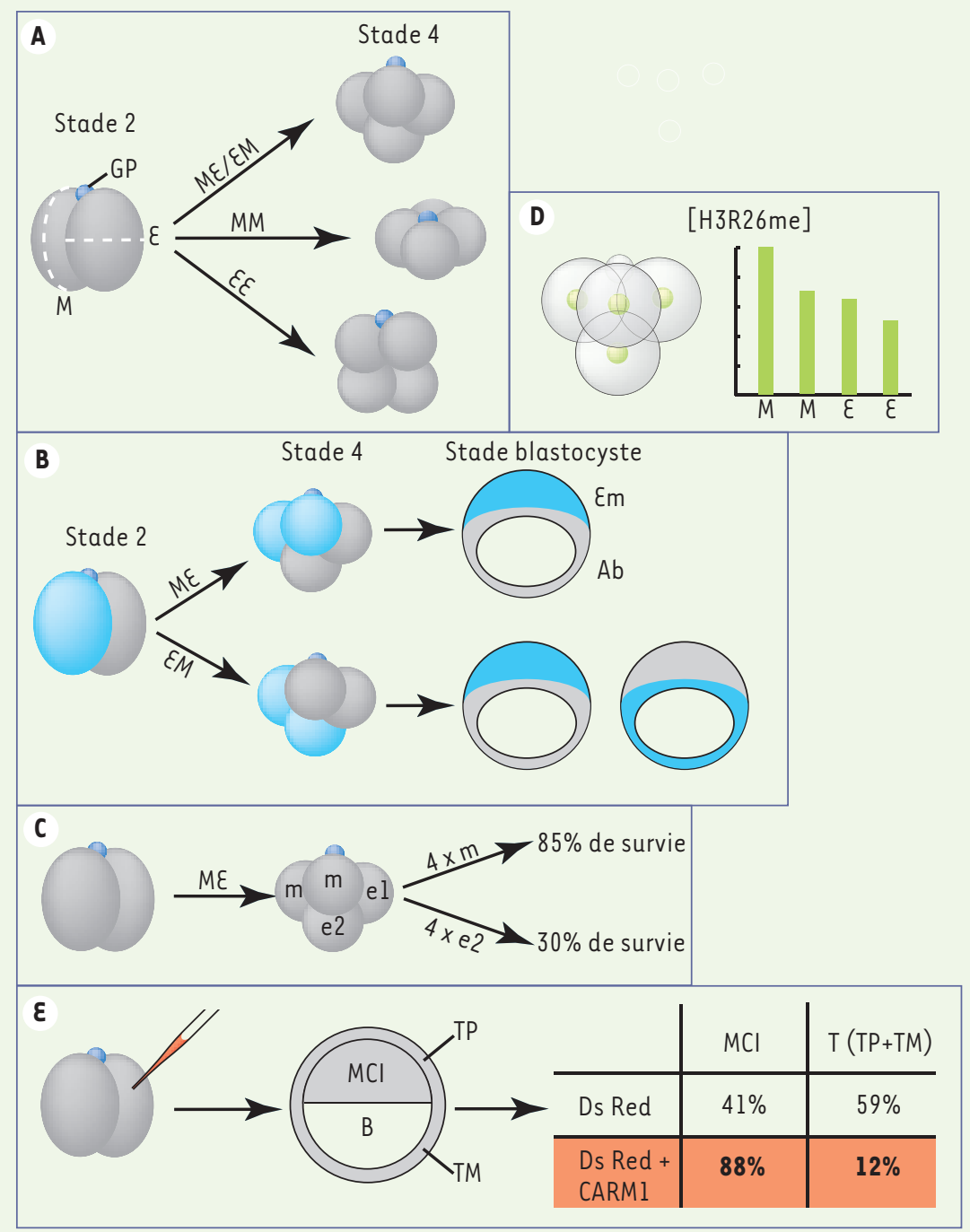

Figure 1. Modifications épigénétiques de la chromatine dans la régulation de la pluripotence des cellules de l'embryon de souris. A. L'origine des trois types de morphologie de l'embryon de souris au stade 4 , de haut en bas, tétraédrique, en étoile, plane. $M$, clivage méridional ; $\varepsilon$, clivage équatorial; gp, deuxième globule polaire. $B$. Relation entre la séquence de clivage des embryons tétraédriques et la contribution des descendants des deux premiers blastomères aux régions embryonnaire $(\varepsilon m)$ et ab-embryonnaire $(A b)$ du blastocyste. C. Capacité des chimères d'agrégation des blastomères $m$ ou e2 à se développer. $D$. Distribution et quantification relative de la marque H3R26me dans un embryon ME. $\varepsilon$. L'injection de la méthyl-transférase CARMI dans un blastomère au stade 2 a pour effet d'augmenter la contribution de ses descendants à la masse cellulaire interne au stade blastocyste. $\mathrm{B}$ : blastocèle; $\mathrm{MCl}$ : masse cellulaire interne ; TP : trophectoderme polaire; TM : trophectoderme mural. et que le niveau de méthylation détecté dans chaque noyau est étroitement corrélé à l'identité du blastomère considéré. Les blastomères e2 présentent ainsi un taux de méthylation qui peut être inférieur de moitié à celui du blastomère $\mathrm{m}$ présentant le taux le plus élevé dans le même embryon (Figure 1D). De façon intéressante, les embryons $\varepsilon M$, dont la polarité ne peut être prédite, présentent dans les noyaux de leurs blastomères des niveaux de méthylation beaucoup plus proches les uns des autres. Au vu de ces résultats, un lien causal a été recherché entre l'activité de méthylation de I'histone $\mathrm{H} 3$ dans un blastomère et le devenir du blastomère en question.

Les arginines 2, 17 et 26 de l'histone $\mathrm{H} 3$ sont les cibles d'une même méthyl-transférase, appelée CARMI. Les auteurs ont montré que chez l'embryon, CARMl est exprimée à partir d'un messager maternel, et qu'il y a une corrélation entre la répartition de la protéine et l'intensité du marquage H3R26me dans les noyaux d'un embryon de stade 4. Afin de tester son impact sur le destin et la potentialité des cellules, I'ARN de CARMI, ainsi que celui du traceur fluorescent DsRed, ont été co-injectés dans un blastomère unique au stade 2. L'analyse des embryons injectés au stade blastocyste a permis de montrer que la descendance des blastomères surexprimant CARMI contribue de façon prédominante à la $\mathrm{MCl}$ (Figure lE). L'utilisation d'une forme mutée de CARMI a confirmé ce résultat en démontrant que c'est bien l'activité méthyl-transférase qui est requise. L'accroissement de l'activité CARMl s'accompagne bien sûr d'une augmentation du marquage H3R26me, mais également d'une expression plus forte des facteurs Nanog et Sox2 qui sont étroitement associés au maintien et à la pluripotentialité des cellules de la $\mathrm{MCl}$.

Cette étude est la première à établir ainsi un lien direct entre la présence d'une marque épigénétique et la détermination d'un destin cellulaire chez un embryon de mammifère. Elle ne permet pas d'expliquer ce qui se passe chez les embryons qui ne subissent pas une séquence de clivage $M \varepsilon$, et dont la plupart se développeront pourtant normalement. Cependant, elle démontre une 
fois de plus que se focaliser sur un cas particulier permet certaines avancées, et suggère une route possible pour de futures investigations. Rappelons qu'à ce jour près de soixante-dix marques épigénétiques sont connues, et en quête de fonctions. De plus, cette étude met en évidence des différences marquées entre les blastomères d'un embryon qui commence à peine à se développer. Nul doute que ces données contribueront au débat très animé que suscite la possibilité d'une régionalisation précoce de l'embryon de souris [4-7]. $\diamond$
Epigenetic regulation of pluripotency in the early mouse embryo

\section{REMERCIEMENTS}

Les auteurs remercient V. Gurchenkov pour la réalisation de la Figure I de cet article.

\section{RÉFÉRENCES}

1. Torres-Padilla ME, Parfitt DE, Kouzarides T, ZernickaGoetz M. Histone arginine methylation regulates pluripotency in the early mouse embryo. Nature 2007; 445: 214-8.

2. Piotrowska-Nitsche K, Perea-Gomez A, Haraguchi $S$, Zernicka-Goetz M. Four-cell stage mouse blastomeres have different developmental properties. Development 2005 ; 132 : 479-90.

\section{NOUVELlE}

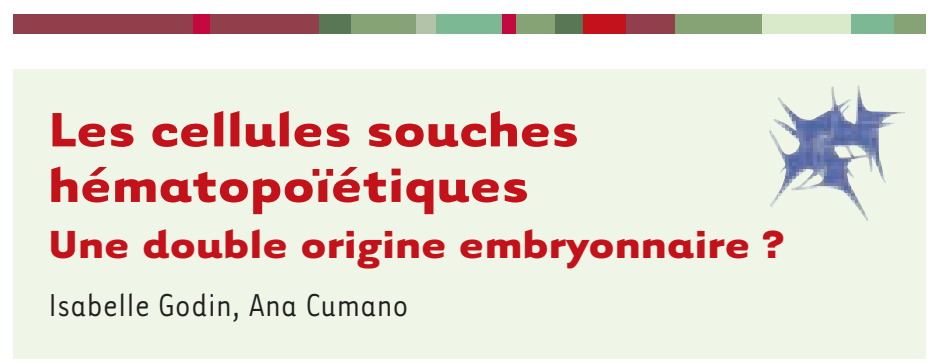

> L'origine des cellules souches hématopoïétiques (CSH) a longtemps été, et reste encore, sujette à controverse. Les CSH de la moelle osseuse adulte sont postulées dériver d'une réserve établie pendant le développement embryonnaire. Initialement, la production du contingent de CSH a été attribuée au sac vitellin (SV), parce que les premières cellules hématopoïétiques, principalement des érythrocytes, apparaissent dans cette annexe embryonnaire.

Cependant, les expériences de chimères entre des corps embryonnaires de caille et des SV de poulet, effectuées avant l'établissement de la circulation entre l'embryon et le SV, ont clairement démontré que les cellules hématopoïétiques du SV ne contribuaient pas à l'hématopoïèse adulte, qui est fondée par des CSH d'origine intraembryonnaire. Plus tard, deux groupes ont montré que, chez la souris également, la génération des cellules hématopoïétiques s'opérait indépendamment dans deux sites (Figure 1).
La première a lieu dans le SV qui produit des cellules érythro-myéloïdes, la seconde prend place plus tard dans le compartiment intra-embryonnaire où la région appelée splanchnopleure para-aortique ( $P-S p)$, puis aortegonades-mésonephros ( $A G M$ ) produit des CSH multipotentes et capables de reconstituer à long terme le système hématopoïétique d'animaux irradiés. Ces observations, aujourd'hui confirmées par plusieurs laboratoires, ont été à la base du concept, généralement accepté, d'une origine des CSH dans le corps embryonnaire, précisément dans la P-Sp/AGM. La quantification des cellules multipotentes a montré qu'elles apparaissaient dans cette région à partir de 8,5 jours de gestation ( $j d g$ ) et qu'elles atteignaient un nombre maximal de 100-150 par embryon, vers 10,5 jdg (35-37 somites), avant de diminuer rapidement en nombre, puis de disparaître vers 12 jdg (pour revue, voir [1]).

Ces expériences ont démontré que, chez la souris, le compartiment intra-
3. Piotrowska-Nitsche K, Zernicka-Goetz M. Spatial arrangement of individual 4-cell stage blastomeres and the order in which they are generated correlate with blastocyst pattern in the mouse embryo. Mech Dev $2005 ; 122: 487-500$.

4. Zernicka-Goetz M. The first cell-fate decisions in the mouse embryo : destiny is a matter of both chance and choice. Curr Opin Genet Dev 2006 ; 16 : 406-12.

5. Hiiragi T, Alarcon VB, Fujimori T, et al. Where do we stand now? Mouse early embryo patterning meeting in Freiburg, Germany (2005). Int J Dev Biol 2006; $50: 581-7$.

6. Louvet-Vallee S, Vinot S, Maro B. Mitotic spindles and cleavage planes are oriented randomly in the two-cell mouse embryo. Curr Biol 2005 ; 15 : 464-9.

7. Kurotaki $Y$, Hatta K, Nakao K, et al. Blastocyst axis is specified independently of early cell lineage but aligns with the ZP shape. Science 2007 ; $316: 719-23$
I. Godin : Inserm U790,

Institut Gustave Roussy-PRI,

39 , rue Camille Desmoulins,

94805 Villejuif, France.

Université de Paris XI, Orsay, France.

A. Cumano : Unité de Développement

des Lymphocytes, Département

d'immunologie, Institut Pasteur,

25 , rue du Docteur Roux,

75724 Paris Cedex 15, France et Inserm U668.

\section{cumano@pasteur.fr}

embryonnaire produisait des CSH. Cependant, la possibilité de la génération tardive d'une fraction de $\mathrm{CSH}$ au niveau du SV ne peut pas être évaluée après $8 \mathrm{jdg}$ (4-5 somites) puisque, à partir de ce stade, la circulation des cellules sanguines dans le réseau vasculaire entraîne un mélange des cellules provenant du SV et de celles provenant du compartiment intraembryonnaire. L'approche de ce problème en utilisant des souris knock in n'est pas possible puisque les facteurs exprimés et/ou impliqués dans la génération des précurseurs hématopoïétiques sont identiques dans les deux sites. Une contribution du SV à I'hématopoïèse adulte demande pourtant à être évaluée puisque des expériences de greffe chez l'embryon d'amphibiens [2] et de souris [3] ont montré une capacité des cellules du SV à engendrer des lymphocytes. 